\title{
In situ cooling with ice water for the easier removal of self-expanding nitinol stents
}

Authors

Institutions
Daniel Merkel ${ }^{1}$, Eckhard Brinkmann², Daniel Wiens ${ }^{1}$, Karl-Michael Derwahl ${ }^{1}$

${ }^{1}$ Clinic for Internal Medicine, St. Hedwig-Krankenhaus, Berlin, Germany

2 Department of Gastroenterology, St. Josefs-Hospital, Cloppenburg, Germany

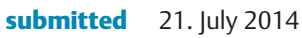
accepted after revision 26. August 2014

\section{Bibliography}

Dol http://dx.doi.org/ 10.1055/s-0034-1390760

Published online: 17.11.2014

Endoscopy International Open 2015; 03: E51-E55

(c) Georg Thieme Verlag KG Stuttgart . New York E-ISSN 2196-9736

\section{Corresponding author}

\section{Daniel Merkel, MD}

Clinic for Internal Medicine

St. Hedwig-Krankenhaus

Grosse Hamburger Strasse 5-11

10115 Berlin

Germany

Fax: +49-30-23112324

merkel@gmx.com
Background: It is yet to be determined what effects temperature has on the properties of nitinol in order to simplify the process of removing nitinol self-expanding metal stents (SEMS).

Materials and methods: We describe the procedure for removal of SEMS in a total of 11 cases with 9 patients. A study involving cooling of nitinol stents in situ with ice water just before their removal was attempted.

Results: All stents were removed successfully. In partially covered and in fully covered stents, the stent rigidity was noticeably reduced following cooling. Stent removal was performed by inver-

\section{Introduction}

$\nabla$

Covered self-expanding metal stents (SEMS) are available in a wide variety of designs, and vary widely with regard to shape, size, materials, coatings, and placement techniques [1,2]. Although their use was initially limited to the palliative care of patients with malignant strictures, SEMS now have a variety of applications, including the treatment of benign strictures, and the management of fistulas and perforations. Furthermore, SEMS have been deployed in the treatment of persistent bleeding [3], as well as being used in the transgastric drainage of pancreatic pseudocysts [4].

With the exception of biodegradable stents (for which the evidence remains inconclusive) [5], SEMS removal should be endoscopically performed because this removal may become necessary when complications occur or if stent treatment is being employed for a benign disorder.

Whereas there are numerous publications on the indications, techniques, and possible complications for stent treatment, only a small number of these are dedicated to exploring the techniques involved in the removal of SEMS [6-9]. Although extraction systems with hooks are generally accepted as the current standard, any endoscopic sion, which was achieved by pulling on the stent from its distal end. No adverse events were observed during this trial.

Conclusion: The higher pliability of the stents after ice-water cooling facilitates stent removal. With this method, a mobilization of all stents by the invagination technique was achieved. The separation of the uncoated stent ends from the intestinal wall by the invagination technique, as well as the mucosal vasoconstriction resulting from the cooling, lead to an easier SEMS removal and may serve to prevent severe bleeding of the mucosal wall during this process.

tools capable of grasping either the stent or the nylon thread should be regarded as possible devices.

Today, more than $90 \%$ of SEMS that are used in the field of gastroenterology are made of nitinol [1]. The term nitinol refers to a group of highly elastic metal alloys of nickel and titanium that can change shape in response to variations in temperature (the so-called shape memory effect) [10]. This type of stent is relatively pliable at room temperature and can therefore be mounted on delivery systems. The higher internal gastrointestinal tract temperature encourages the stent to resume its preformed shape. This characteristic determines the stent's radial force and consequently its therapeutic effect.

The fact that the stent's rigidity is dependent upon temperature can be utilized for an easier process of mounting the stent on the delivery system [11]. The in situ use of ice water for easier implantation has been described with regard to vena cava filters [12]. However, it remains to be determined whether the physical properties of nitinol might also allow the uncomplicated removal of stents. 


\section{Materials and methods}

In a small in vitro experimental study, we were able to show that ice-water cooling leads to nitinol stents becoming less rigid, with the effect becoming apparent immediately upon irrigation ( $\bullet$ Fig. 1).

Stent removal following cooling with ice water was performed on a total of 9 patients, and involved 11 cases with nitinol SEMS. Of these, 3 were esophageal, 5 cardial, 2 pyloric, and 1 rectal $(\bullet$ Table 1 ). In all cases, the decision to perform stent removal was based on clinical need alone, and was not dependent upon participation in this study. All the procedures were performed in our primary care hospital's gastroenterology department and were part of the department's normal clinical routine.

All patients involved were provided with detailed information about the study and gave written consent prior to undergoing stent removal. The local institutional review board approved the study protocol.

All procedures were performed using intravenous (IV) sedation with propofol, and involved continuous patient monitoring. All endoscopic examinations were performed using standard highresolution gastroscopes (Olympus, Hamburg, Germany) and included an inspection of the stent's distal end as well as the sections beyond. A spray catheter (Endo-Flex, Voerde, Germany), which is normally used in preparing for radiofrequency ablation (RFA) therapy, was introduced via the endoscope's instrument channel just prior to stent mobilization. This was used to spray the stent in a uniform manner from the luminal side using 100 to $160 \mathrm{~mL}$ of ice-cooled tap water $\left(4^{\circ} \mathrm{C} / 39.2^{\circ} \mathrm{F}\right)(\bullet$ Fig. 2$)$. The water was manually applied using a standard $10 \mathrm{~mL}$ syringe.

Standard rat-tooth forceps (Endo-Flex, Voerde, Germany) were used to grasp either the nylon thread, or the wire mesh on the distal end of the stent.

Once the stent had been removed, the endoscope was re-inserted in order to check for potential complications, in particular acute mucosal hemorrhage. All patients were closely monitored for at least 24 hours following stent removal.

\section{Results}

$\nabla$

All stents were successfully removed without complications. In all cases, forceps were able to capture the nylon thread or the wire mesh at the distal end. Stent mobilization was preceded by inversion, which was achieved by pulling on the stent's distal end (๑ Fig. 3).

The process of stent cooling with ice water varied according to stent type:

- Uncovered stents $(n=2)$ : Most of the stent's wire mesh was covered by hyperplastic mucosal overgrowth, and no relevant cooling effect was detected in relation to the wire mesh.

- Partially covered stents $(n=7)$ : Stent rigidity was noticeably reduced following cooling. Stent diameter was noticeably decreased inside the coated section, leading to sections of the stent detaching from the mucosa and a complete loss of the stent's radial force.

- Fully covered stents $(n=2)$ : Stent diameter decreased noticeably in response to cooling. This resulted in a reduction of the stent's radial force (i.e., its ability to push outward against the mucosal tissue). The stent's reduced rigidity resulted in easier removal, with no significant resistance being observed. Due to the wire frame being fully covered, the stent ends were affec-
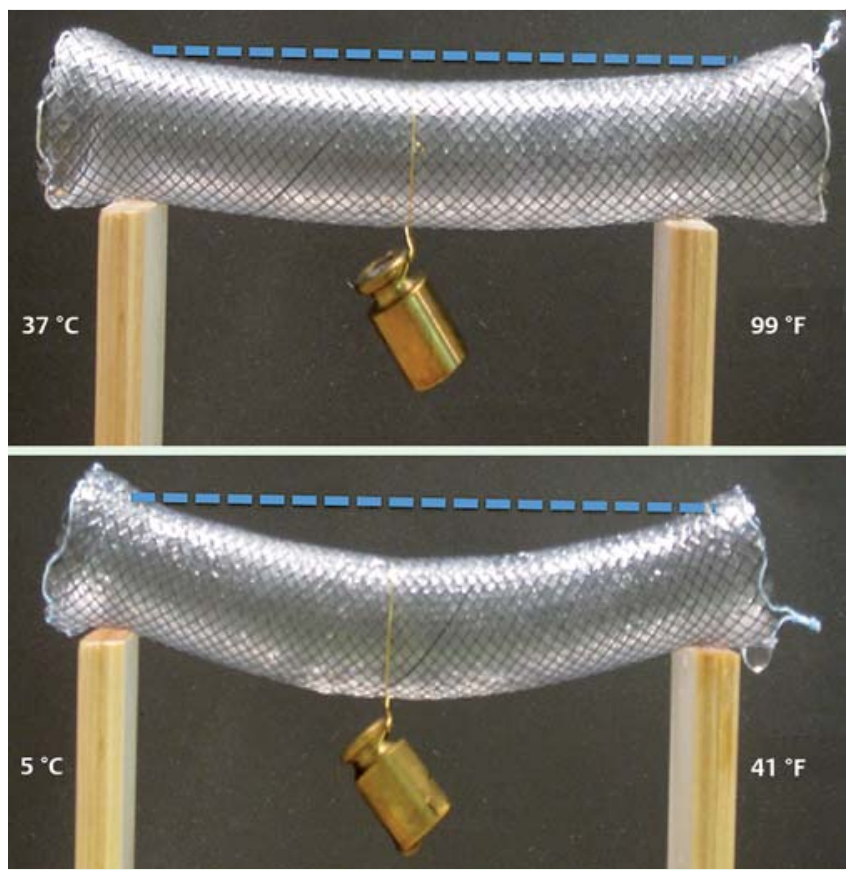

Fig. 1 An in vitro experiment. Immediately after application of ice water to the stent, the rigidity of the stent decreases and the pliability increases.

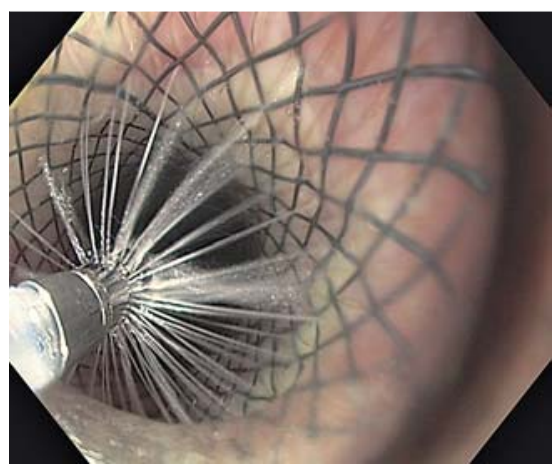

Fig. 2 A spray catheter is used to apply ice water to the stent in a uniform manner from the luminal side.

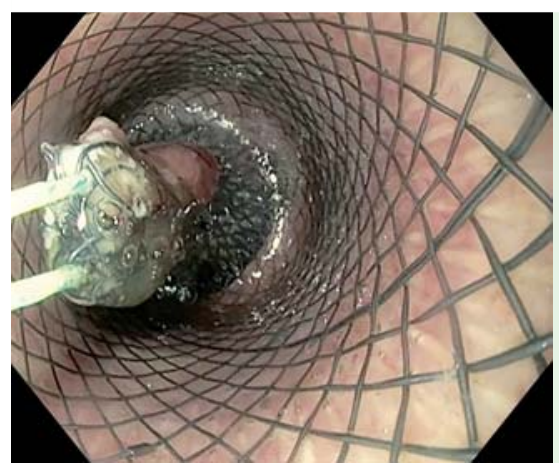

Fig. 3 Mobilizing of the stent using the inversion technique achieved by pulling on the stent from its distal end.

ted by only minor mucosal overgrowth and stent removal did not result in significant mucosal bleeding.

- Grasping hold of their distal ends successfully captured all the stents. They were then mobilized by inversion, and removed in a retrograde fashion. Stent inversion resulted in the detachment of uncovered stent ends from the mucosal tissue and cooling resulted in vasoconstriction within the tissue. This resulted in only moderate and self-limiting bleeding. 


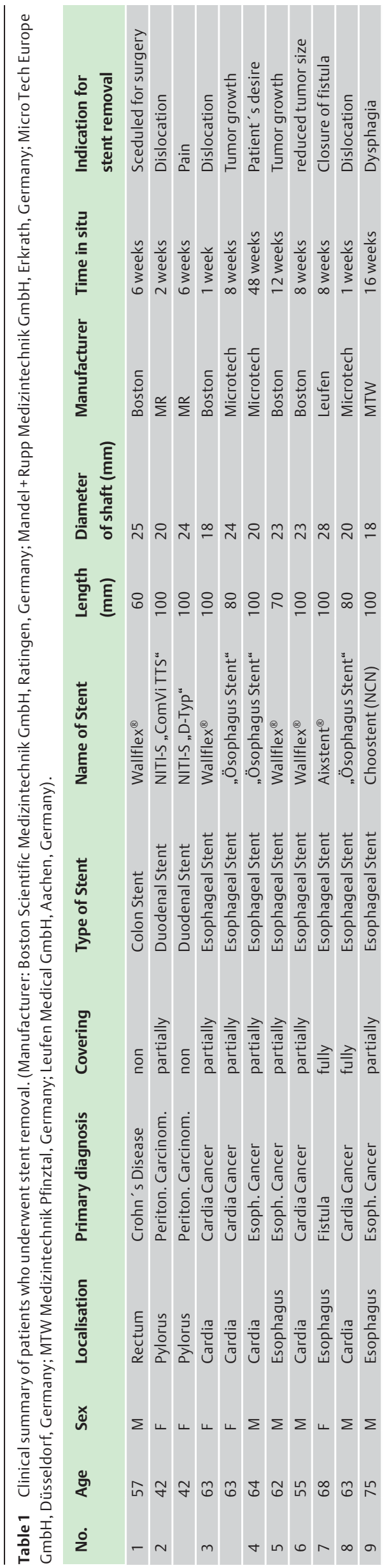

\section{Discussion}

Due to the sheer variety of available stent designs, differences were expected regarding the outcomes of stent removal. Uncovered stents, for instance, are known to show significant levels of mucosal overgrowth between stent struts. Although this tissue overgrowth helped to secure the stent [9], it can lead to difficulties should the stent need to be removed at a later date. In contrast, it is rare for undamaged, fully covered stents to be affected by significant levels of mucosal overgrowth [9]. This makes endoscopic removal of the stent comparatively easy, but also increases the rate of stent dislocation [7]. Our clinic predominantly uses what constitutes a compromise between uncovered and fully covered stents-that is, stents that have a covered shaft but whose flared ends remain uncovered. In 3 of the 11 cases described in this report, stent dislocation was the primary reason for stent removal.

Most SEMS have no explicit approval for stent removal. Therefore, their removal has had to be performed in an unconventional fashion that may be associated with uncertain risk and possible complications. In Europe, only a few of the fully covered stents have approval for retrieval. In our case series, fully covered stents, which are approved for stent removal, have been used only in two patients (see Table 1, patients 7 and 8; cases 9 and 10). In all other cases, the stent retrieval had to be performed due to complications, or the fact that the stent had not been available in the desired size (patient 1, case 1).

Only a small number of case series are available that have dealt with either the techniques involved in the removal of SEMS or the safety of removal procedures [13-17]. In the largest case series to date, van Halsema et al. reported about the safety of endoscopic removal of SEMS in the treatment of benign esophageal diseases in a total of 329 multicenter stent removals. The overall success rate for endoscopic stent removal was very high, with a rate of $8.5 \%$ minor adverse events and $2.1 \%$ major adverse events [18].

The temperature-dependent characteristics of nitinol indicate that the material is ideally suited for use in stenting [10]. Whereas numerous in vitro studies have attested to the fact that an increase in temperature leads to an increase in the material's rigidity, only a small number of articles have described the reverse effect. Song et al. reported on the process of cooling SEMS prior to mounting on a delivery system [6]; and there has been reporting on some of the potential clinical uses of this technique in the field of vascular medicine [12]. The cooling of nitinol stents before retrieval has been previously documented in a clinical case report of patients with benign prostatic hyperplasia [19] and tracheal stenting in animals [20]. No studies have been published regarding how the material's temperature-dependent characteristics might be utilized for the process of stent removal in the gastrointestinal tract.

Although it is possible to remove a stent by grasping its proximal end (either by capturing the removal thread or the stent's wire mesh), our experience suggests that removal by inversion-turning the stent inside out by pulling on it from its distal end-is associated with greater benefits. This process allows the stent to be lifted away at a $90^{\circ}$ angle rather than by being pulled along the tissue layer, minimizing shearing forces and reducing the amount of tissue damage caused by the removal of stent sections overgrown with mucosal tissue. Once the process of inversion has started, and the stent's distal end has been folded inward, the process of mobilization is gradual, with the stent being pulled 
away from the surrounding tissue section by section rather than all at once. This requires only a marginally increased level of traction to be applied via the endoscope. Whereas our experience shows that this process is gentler on the patient, the nature of the procedure means that the stent blocks the scope's view throughout the procedure (similar to stent removal from the proximal end), making it impossible to inspect the damage caused to the mucosal tissue. This results in re-insertion of the endoscope in order to assess the extent of any acute complications.

In fully covered and partially covered stents, the process of spraying the inside of the stent with ice water led to the desired results, with all stents exhibiting an immediate and marked decrease in rigidity. The diameter of the stents decreased and the covering membrane showed folds ( $\bullet$ Fig. 4 and $\bullet$ Fig.5). A gap between the stent and the esophagus wall was also visible (๑ Fig. 6).

In uncovered stents, there is normally tissue growth between the stent meshes. Thus the cooling process was not sufficient to separate the uncovered sections of the stent from the mucosa. Therefore, we see no convincing advantages to cooling uncovered SEMS before removal.

For partially covered stents, a combination of the processes described above was observed. The wire meshes of the uncovered section were often not overgrown with mucosa over the entire circumference. We observed that the remaining visible portion of the mesh, after cooling, was elevated from the mucosa and therefore could be easily gripped by the forceps. Once this was achieved, the stent could be withdrawn.

It is generally accepted that the use of iced saline has no advantages in the treatment of gastric ulcer bleeding. However, ulcer bleeding is due to a different mechanism than that of mechanical tissue damage due to stent removal. Because the process of cooling causes temporary vasoconstriction in the mucosal tissue, it is possible that any bleeding caused by the removal of impacted stent sections might have been less severe than without cooling. However, none of our patients required endoscopic therapy to stop bleeding immediately after stent removal; nor did any of our patients go on to develop significant bleeding complications requiring interventions. Because this was only a small case series, the conclusion cannot be drawn that ice-water cooling significantly reduced the risk of bleeding. Further studies of this nature with a larger patient sample size are required to verify these findings.

The longer a stent is in situ, the harder it will be to remove. Gouveris et al. reported on a case involving a partially covered esophageal stent that was removed 11 months after initial implantation using a rigid endoscope [21]. Our case series included one patient with a partially covered esophageal stent that had been in situ even longer (12 months), and which we were able to remove using a flexible endoscope and the technique of stent inversion described above. The stents included in our case series had been in situ for an average duration of 11 weeks (minimum 1 week, maximum 12 months).

Given that drinking copious amounts of ice water achieves the same effect as cooling with ice water, it would appear obvious that the use of ice water to cool the upper gastrointestinal tract must be relatively well tolerated, at least in the proximal esophagus. There is of course no denying the fact that the application of ice water can lead to a vagal response. Despite continuous monitoring, we observed no such response in any of our patients; nor did we observe esophageal spasms or any other adverse reactions

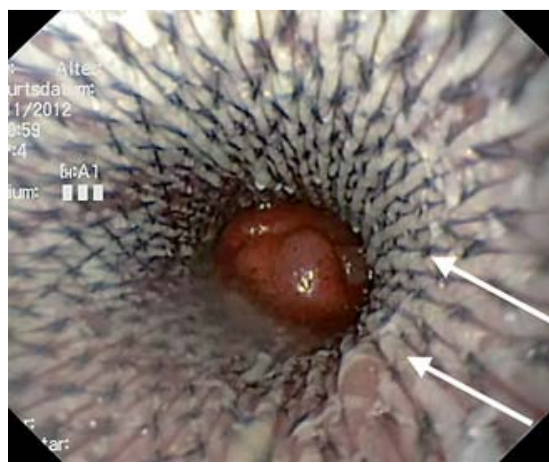

Fig.4 Immediately after ice-water cooling, the covering membrane shows creases (arrows) as a result of decreasing the diameter of the stent (patient 2, case $3 ; 1$ week after stent placement).

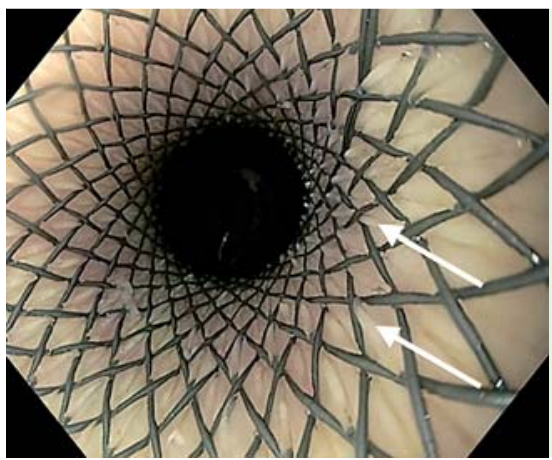

Fig. 5 The same effect as shown in Fig. 4 occurred in patient 9 , case 11 (arrows). The stent was removed 16 weeks after placement.

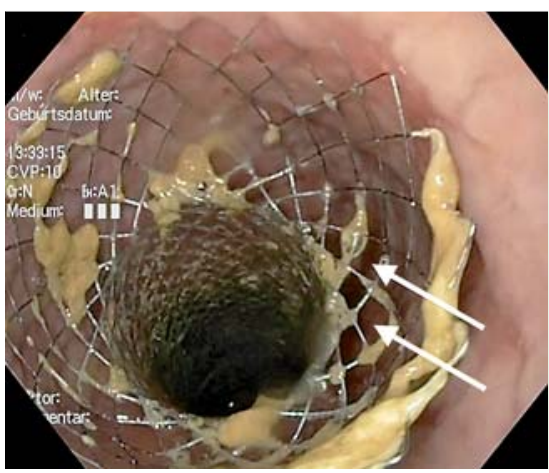

Fig. 6 Sometimes a gap (arrows) between the stent and the esophagus wall shows the decreased diameter of the stent after icewater cooling (patient 5 , case 7).

affecting the esophagus. Effective sedation of patients prevented us from ascertaining any potential pain response.

Using a spray catheter to apply ice water is a simple and straightforward procedure that can easily be performed by auxiliary staff. In our case series, a minimum of $100 \mathrm{~mL}$ of ice water had to be applied in order to achieve a discernible effect. A maximum limit was imposed because the risk of aspiration increases with the amount of water applied. An additional risk of aspiration can be assumed if the stent therapy takes place in the upper half of the esophagus. In our case series we had two such cases (patients 5 and 9; cases 7 and 11), in which no visible aspiration occurred. Nevertheless, the risk of aspiration should not be underestimated.

This procedure also requires a certain level of dexterity because the removal of the spray catheter from the instrument channel and the insertion of a suitable tool for grasping the removal thread must be accomplished before the effects of the cooling process wear off.

Only a few SEMS are designed with a thread on the distal end. Such a thread facilitates the correct positioning immediately following stent implantation. We are not aware of any stents that have a thread attached to the distal end that explicitly allows stent retrieval in the inversion technique. 
With regard to the limitations of this study, a number of factors must be noted that affect the applicability of results: limited number of cases, single-center study, and lack of randomization. The main limitation, however, is the fact that we only included patients with strictures wide enough to be passed by an endoscope. Stents with a smaller diameter (e.g., biliary stents) and stents used for the treatment of severe strictures are sometimes more difficult to remove. They are usually not removed via the invagination technique, although ice-water cooling seems to be feasible when the spray catheter can pass through the stenosis or there is a small stent diameter, respectively.

\section{Summary \\ $\nabla$}

In situ cooling with ice water results in a marked reduction in the rigidity of the nitinol stent. As expected, this effect was particularly pronounced in fully covered and partially covered stents. The resulting increased pliability of the stents made it possible to remove the stents by capture and inversion of the distal end. Using this process, only minimal and self-limiting mucosal wall bleeding after the stent removal was observed.

In our experience, the ice-water cooling of nitinol stents facilitates the uncomplicated process of stent retrieval.

This is the very first description of in situ cooling with ice water to ease the removal of self-expanding nitinol stents. There were no significant complications in this series of cases. However, it is not possible to make a definitive statement on the safety of this method due to the small number of cases in this study.

\section{Competing interests: None}

\section{References}

1 Sharma P, Kozarek R. Practice Parameters Committee of the American College of Gastroenterology. Role of esophageal stents in benign and malignant diseases. Am J Gastroenterol 2010; 105: 258 - 273

2 Hirdes MMC, Vleggaar FP, de Beuile M et al. In vitro evaluation of the radial and axial force of self-expanding esophageal stents. Endoscopy 2013; 45: 997-1005

3 Herzog J, Eickhoff A. Selbstexpandierende metallstents im gastrointestinaltrakt. Der Gastroenterologe 2012; 7: 435-449

4 Eickhoff $A$. Self-expandable metal stents in the gastroenterology tractindications and fields of application. Endo heute 2013; 26: 150-160
5 Repici A, Vleggaar FP, Hassan C et al. Efficacy and safety of biodegradable stents for refractory benign esophageal strictures: the BEST (Biodegradable Esophageal Stent) study. Gastrointest Endosc 2010; 72: 927-934

6 Song HY, Park SI, Jung HY et al. Benign and malignant esophageal strictures: treatment with a polyurethane-covered retrievable expandable metallic stent. Radiology 1997; 203: 747-752

7 Eloubeidi MA, Talreja JP, Lopes TL et al. Success and complications associated with placement of fully covered removable self-expandable metal stents for benign esophageal diseases. Gastrointest Endosc 2011; 73: 673-681

8 Chang Yoon CJ, Shin JH, Song HY et al. Removal of retrievable esophageal and gastrointestinal stents: experience in 113 patients. AJR Am J Roentgenol 2004; 183: $1437-1444$

9 Kozarek R, Baron T, Song HY (eds.) Self-expandable stents in the GItract. New York: Springer Science+Business Media; 2013

10 Stoeckel D, Pelton A, Duerig T. Self-expanding nitinol stents: material and design considerations. Eur Radiol 2004; 14: 292 - 301

11 Song HY, Jung HY, Park SI et al. Covered retrievable expandable nitinol stents in patients with benign esophageal strictures: initial experience. Radiology 2000; 17: $551-557$

12 Duerig T, Pelton A, Stöckel D. An overview of nitinol medical applications. Materials Science and Engineering 1999; 273: 149-160

13 Song $H Y$, Kim SB, Jung HY et al. Retrievable expandable nitinol stents: experiences in 108 patients with esophagogastric neoplasms. J Vasc Interv Radiol 2002; 13: 285-293

14 Park JH, Song H-Y, Kim JH et al. Polytetrafluoroethylene-covered retrievable expandable nitinol stents for malignant esophageal obstructions: factors influencing the outcome of 270 patients. AJR Am J Roentgenol 2012; 199: 1380-1386

15 Thomas T, Abram KR, Subramanian Vet al. Esophageal stents for benign refractory strictures: a meta-analysis. Endoscopy 2011; 43: 386-393

16 Talreja JP, Eloubeidi MA, Sauer BG et al. Fully covered removable nitinol self-expandable metal stents (SEMS) in malignant strictures of the esophagus: a multicenter analysis. Surg Endosc 2012; 26: 1664-1669

17 Suntharanathan J, Edwards DW, Mullan D et al. New "knitted" egis esophageal stent allows atraumatic inside-out removal by inversion. Endoscopy 2013; 45: E187-E188

18 van Halsema EE, Wong Kee Song LM, Baron TH et al. Safety of endoscopic removal of self-expandable stents after treatment of benign esophageal diseases. Gastrointest Endosc 2013; 77: 18-28

19 Marks LS, Ettekal B, Cohen MS et al. Use of a shape-memory alloy (nitinol) in a removable prostate stent. Tech Urol 1999; 5: 226-230

20 Vinograd I, Klin B, Brosh T et al. A new intratracheal stent made from nitinol, an alloy with "shape memory effect". J Thorac Cardiovasc Surg 1994; 107: 1255-1261

21 Gouveris HT, Mann WJ, Lippert BM. Successful rigid endoscopic removal of an esophageal subtotally covered nitinol stent 11 months after initial placement. Eur Arch Otorhinolaryngol 2009; 266: 927-931 